\title{
A novel method to establish the rabbit model of knee Osteoarthritis: Intra-articular Injection of SDF-1 induces $O A$
}

\section{CANZHANG LI}

Departments of Sports Medicine, The First Affiliated Hospital, Kunming Medical University, Kunming, Yunnan

\section{YINHONG HE}

Departments of Sports Medicine, The First Affiliated Hospital, Kunming Medical University, Kunming, Yunnan

\section{YANLIN LI ( 852387873@qq.com )}

Departments of Sports Medicine, The First Affiliated Hospital, Kunming Medical University, Kunming, Yunnan

\section{GUOLIANG WANG}

Departments of Sports Medicine, The First Affiliated Hospital, Kunming Medical University, Kunming, Yunnan

\section{DEJIAN LIU}

Departments of Sports Medicine, The First Affiliated Hospital, Kunming Medical University, Kunming, Yunnan

\section{GUOFENG CAI}

Departments of Sports Medicine, The First Affiliated Hospital, Kunming Medical University, Kunming, Yunnan

\section{CHUAN HE}

Departments of Sports Medicine, The First Affiliated Hospital, Kunming Medical University, Kunming, Yunnan

\section{Research Article}

Keywords: osteoarthritis, intra-articular injection, SDF-1, animal model

Posted Date: November 12th, 2020

DOI: https://doi.org/10.21203/rs.3.rs-103520/v1

License: (c) (1) This work is licensed under a Creative Commons Attribution 4.0 International License. Read Full License 
Version of Record: A version of this preprint was published at BMC Musculoskeletal Disorders on April 3rd, 2021. See the published version at https://doi.org/10.1186/s12891-021-04188-7. 


\section{Abstract}

\section{Background}

Animal model of Knee Osteoarthritis $(\mathrm{OA})$ is the primary testing methodology for studies on pathogenic mechanisms and therapies of human OA disease. Recent major modeling methods are divided into artificially induced and spontaneous. However, these methods have some disadvantages of slow progression, high cost and no correlation with the pathogenesis of OA.

\section{Methods}

Our studies attempted to find a rapid, easy, and consistent with the natural pathological process of OA modeling method by intra-articular injection of SDF-1 (stromal cell-derived factor 1, a chemokine naturally occurring during the pathological process of human $\mathrm{OA}$ ) in the rabbit knee. After induction we collected cartilage specimens from the medial femoral condyle to undergo macroscopic, histological, immunohistochemical, and biochemical evaluations. Meanwhile, compared with Hulth surgical method to evaluate its efficacy.

Results

Macroscopic observation and Mankin's score of histological staining exhibited typical features of middle stage OA cartilage in SDF-1 injected groups. Immunohistochemically, the positive expression of IL-1 (interleukin-1) and TNF-a(tumor necrosis factor a) was earlier and higher in high dose SDF-1 group than surgical group. The levels of matrix metalloproteinases (MMPs) in synovial fluid and in chondrocytes significantly increased, and type II collagen (COLX) and aggrecan (ACAN) expressions decreased in SDF-1 injected group following the extension of time and increase of SDF-1 concentration.

\section{Conclusions}

Our data indicated intra-articular injection of SDF-1 (40ug/ $\mathrm{kg}$, three times for 12 weeks) can induce rabbit knee OA model successfully, more rapidly and easily than traditional surgical modeling. The study provides a further option for the establishment of knee OA animal model.

\section{Background}

Knee Osteoarthritis (OA), a most common joint disorder in the elderly, which is characterized include joint degeneration, loss of cartilage, and alterations of subchondral bone. It is the most common form of arthritis, with the highest morbidity rate of any illness. More than 40 million Americans have OA, including $80 \%$ of persons older than 50 years. [1] The prevalence rate of knee osteoarthritis in Chinese aged over 40 years old reached 15.6\%. [2] The causes of OA are unknown, but it is reported that obesity, bone mass, joint injury, instability, trauma, joint deformity and age are common factors in OA, especially in the hip and knee joints. [3] Many studies have suggested that OA affects many joint structures, including degeneration of cartilage, abnormal bone remodeling and synovial inflammation, which pathobiology is 
far more complex. [4] To explore the pathobiology of OA, early detection and intervention are important roles to prevent and treat OA.

Animal models research is an important method to study OA pathology, included artificially induced or spontaneous models. Spontaneous model which has naturally occurring and genetically modified models that develop OA is the perfect approach similar to the OA pathological process, but it requires a long modeling period and high cost. [5, 6] Compared with spontaneous models,artificially induced modeling has a rapid progression, common methods including biochemically or surgically induced. Among them, surgical modeling has a high induction rate and good modeling stability, but it has a great impact on the biochemistry and high risk of infection of joints due to surgical trauma and joint instability. Hulth surgical modeling is the most classic and popular used method.[7] Another modeling method, intraarticular injection chemicals such as papain and collagenase, shows high results in relatively homogeneous degree and reproducibility of a pathological state in a short period. However, the method exists controversy about the pathophysiology, which biochemical model might induce significant amount of inflammation and also may not represent naturally occurring OA state found in human. [8]

Stromal cell derived factor-1 (SDF-1), produced by synovial cells in OA joint, is a cytokine that is associated with inflammation. Once SDF- 1 binds to its ligand C-X-C chemokine receptor type 4 (CXCR4), a $G$ protein-coupled receptor located in the surface of chondrocytes, it activates multiple downstream signaling pathways to induce chondrocyte apoptosis and release MMPs to degrade extracellular matrix (ECM) causing cartilage degradation and increasing the pathological process of OA.[9-11] Some studies showed that the SDF-1/CXCR4 signaling pathway plays a critical role in the pathogenesis of OA cartilage degeneration and a remarkable increase of SDF-1 in synovial fluid from patients with OA. [11, 12] Xu et al. demonstrated that the SDF-1 level was associated with the radiographic severity of OA. [13] Our previous studies found the use of CXCR4 inhibitors AMD3100 and TN14003 could block the SDF-1/CXCR4 axis and reverse the OA process. $[12,14]$

Therefore, it was assumed that exogenous SDF-1 could be used to simulate the pathological process of $\mathrm{OA}$, and establish the OA animal model. Our experiment tries to use SDF-1 intra-articular injection to induce $O A$ animal models, explore the appropriate dose and time for modeling and compare with Hulth surgical modeling in morphology, histology and cartilage matrix composition in order to find a modeling method more similar to the natural OA pathological process.

\section{Results}

\section{Gross observation}

After 4 weeks of injection, there was no significant difference in cartilage surfaces morphology between the experimental groups and the blank group. (Fig. 1A-E). Compare with blank group the cartilage surface of medial femoral condyles in the experimental groups showed roughness and dry, the gloss decreased, and even some cartilage defects appeared in SDF-1 40ug/kg group and surgical group after 8 weeks (Fig. $1 \mathrm{~F}-\mathrm{J}$ ). The changes were even more pronounced after 12 weeks. In 20ug/kg, 40ug/kg SDF-1 group 
and surgical group, joint deformation and cartilage defection were extreme and osteophytes formed at the joint margins (Fig. 1K-0). In various concentrations of SDF-1 injected groups, the severity of cartilage degeneration demonstrates in a dosage dependent manner, SDF-1 40ug/kg group was similar to the surgical group.

\section{Histological evaluation and Mankin score}

After 4 weeks, HE staining in the experimental groups demonstrated slightly cells swelling, the polarity disappearing, the structural disorganization and the number of chondrocytes significantly decreased with a ragged edge (Fig. 2A B-E) compared with blank group (Fig. 2A A). After 8 weeks, the above phenomenon was even more obvious, cartilage surface thinness and deep fissures were observed (Fig. 2A F-J). After 12 weeks, chondrocytes of experimental groups were partly lost or arranged in clusters, cartilage defects were detected, and hypertrophic chondrocytes and deep cracks were visible, especially in the surgical group and SDF-1 40ug/kg group. Damage to articular surface integrity was more significant in the surgical group than in the SDF-1 injection groups (Fig. 2A K-0). The Mankin score was significantly higher in the SDF-1 groups than in the blank group after 8 and 12 weeks. At 12 weeks, Mankin score of SDF-1 $40 \mathrm{ug} / \mathrm{kg}$ group reached the middle stage of $O A$ [15] which is closed to the surgical group with statistical difference (Fig. 2B).

\section{Immunohistochemical evaluation}

IL-1 and TNF-a immunohistochemical staining were performed to evaluate secretion levels of inflammatory factors in cartilage tissue. It's not obvious at 4 weeks (Fig. 3A A-E,4A A-E), but after 8 weeks the expression levels of IL-1 and TNF-a in the experimental groups were significantly increased in cartilage tissue following SDF-1 injection and surgery. Chondrocytes presented that the polarity disappeared, the structure disorganized and arranged in clusters (Fig. 3A F-J,4A F-J). At 12 weeks the EMC staining was uneven, resulting in cracks and severe damage (Fig. 3A K-0,4A K-0). At 4 weeks, the positive expression of IL-1 and TNF-a in the surgical group was higher than that of the SDF-1 $10 \mathrm{ug} / \mathrm{kg}$ and $20 \mathrm{ug} / \mathrm{kg}$ groups, but close to that in the SDF-1 40ug/kg group $(P>0.01)$. After 8 and 12 weeks, they were lower than those in the SDF-1 40ug/kg group $(P<0.01)$ (Fig. 3B, 4B).

\section{Elisa of MMP-3, 9, 13 levels in synovial fluid}

ELISA revealed SDF-1 injection significantly elevated the content of MMP-3, 9, 13 in the synovial fluid which is catabolic proteases associated with $\mathrm{OA}$ and the main disruptive factors in cartilage degeneration. In the three concentration SDF-1 injected groups and the surgical group, the level of MMP-3, 9,13 in the synovial fluid increased with the extension of time $(p<0.01)$. At the same time point, the level of MMP-3, 9, 13 in the three SDF-1 injection groups increased with the increase of drug administration concentration $(p<0.01)$. Compared with the surgical group, the content of MMP-3, 9,13 in the SDF-1 $20 \mathrm{ug} / \mathrm{kg}$ group and the SDF-1 $40 \mathrm{ug} / \mathrm{kg}$ group at the same time point was higher than that in the surgical group $(P<0.01)$. However, MMP-13 concentration in the synovial fluid increased to a certain level after 8 weeks, the growth rate gradually slow $(P>0.01)$ (Fig. 5A, 5B, 5C).

MMP3,9,13 mRNA expression levels in cartilage of OA model rabbit. 
The expression levels of MMP-3, 9, 13 mRNA in articular cartilage at 4,8 and 12 weeks after treatment was determined by qRT-PCR. The MMP-3, 9, 13 mRNA expression levels in the surgical group and the three SDF-1 dose groups were all increased with the extension of time compared with the blank group ( $p$ $<0.01$ ). After 12 weeks, MMP-3, 9, 13 genes expression in the SDF-1 20ug/kg and 40ug/kg groups was superior to those in the surgical group. Results associate with Elisa showed that the expression of MMP$3,9,13$ genes and protein secretion could be induced more rapidly with high dose of SDF-1 injection than Hulth operation group. After 8 weeks, MMP-13 mRNA expression in the SDF-1 40ug/kg group increase was not obvious similarity with Elisa test $(P>0.01)$. (Fig. 6A,6B,6C) It may indicate that the modeling experiment only went to the middle stage of $\mathrm{OA}$ which consistent with previous research conclusions, MMP-13 is abnormally expressed in different stages of the OA process which is up-regulated in the early stage of OA cartilage and down-regulated in the late stage. [16]

\section{The effect of SDF-1 on COL $\mathbb{Q} \square A C A N$ in chondrocytes and cartilage}

To evaluate the effect of SDF-1 induced OA model, we investigated the expression level of COLХIACAN in chondrocytes and cartilage which are the main structural component of cartilage using QPCR and western blot analysis.

QPCR demonstrated the expression level of COL\mRNA and ACAN mRNA in the blank group did not change significantly with the extension of time, but they decreased with the extension of time in the experimental group $(p<0.01)$. At the same time point, the mRNA expression level of COL\and ACAN in the different concentration SDF-1 injected groups reduced with the increase of drug concentration $(p<0.01)$. At 12 weeks, the mRNA expression level in the SDF-1 injected groups are reduced without a significant difference compared with the surgical group $(p>0.01)$ (Fig. 7A,7B).

Western blot analysis showed COL『and ACAN protein expressions diminished over time in the experimental group $(p<0.01)$. SDF-1 induced decreased COL『and ACAN protein expressions in a dosedependent manner $(p<0.01)$. Our experiment revealed the COL $\otimes$ and ACAN degradation gradually along with the progress of osteoarthritis, and SDF-1 accelerated the process. Moreover, at the same time point

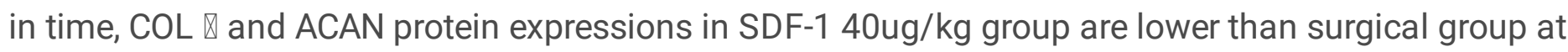
$4,8,12$ weeks $(p<0.01)$. It suggested that the SDF-1 injection compared with Hulth surgery, the loss of $\mathrm{COL} \otimes \mathrm{ACAN}$ and cartilage damage is more. (Fig. 8A, 8B)

\section{Discussion}

Although, extensive animal models for OA study have been developed over the past half century, there is no gold standard animal model used in OA research. In the ideal animal model, the disease must be induced reliably, with $100 \%$ penetrance, and within a suitable time frame, and yet still present with disease characteristics that are comparable to the human condition. $[17,18]$ As an important chemokine produced in the pathological process of human OA, we found that intra-articular injection of SDF-1 as a novel biochemical method can successfully induce knee OA in rabbits, and the effect with SDF-1 40ug/kg 
injection after 12 weeks is similar to the classic surgical modeling. This modeling method may be another choice closer to the natural OA pathology.

Macroscopic observation and HE staining of SDF-1 treated group in our experiment showed typical characteristics of early OA described in the literature [19], and Mankin score indicating a histological improvement of cartilage deterioration by SDF-1 and the degeneration of SDF-1 $40 \mathrm{ug} / \mathrm{kg}$ group on articular cartilage went to middle stage of $O A$ at 12 weeks that was similar to the surgical group. This result is also consistent with our previous research.[20]

As pivotal pro-inflammatory cytokines in AO pathology, IL-1 and TNF-a play roles in promoting cartilage matrix degradation. $[21,22]$ In the immunohistochemical study, the positive expression of IL-1 and TNF-a are significantly increased in all SDF-1 groups, and uneven extracellular matrix staining, cracks forming and cartilage defects are obvious. After 8 weeks, the expression level of IL-1 and TNF-a in SDF-1 40ug/kg group was higher than the surgical group. It indicated high dose SDF-1 intra-articular injection promotes inflammatory factors secretion earlier than Hulth surgery modeling, and inflammation was more severe. Wang et al. also demonstrated that activation of SDF-1/CXCR4 pathway significantly promoted the secretion of IL-1 $\beta$, IL-6, IL-15, IL-17, IL-18, and TNF-a. These inflammatory factors activate MAPKs pathway and TLRs pathway to promote the apoptosis of chondrocytes, and finally lead to the further imbalance of homeostasis in the knee joint. [23]

MMPs play a key role in the degradation of cartilage matrix and damages the stability of articular cartilage, especially MMP-3,9,13. [24-26] MMPs are rarely expressed under normal physiological conditions, however significantly overexpressed in synovial fluid and articular cartilage of human OA. [27] We examined the levels of MMP-3,9,13 in synovial fluid and the expressions of MMPs mRNA in chondrocytes. Either in joint fluid or chondrocytes, the mRNA expression and secretion levels of the MMP3,9,13 increased with SDF-1 injection concentration and time, indicating that the modeling was successful. At 12 weeks, the content of MMP-3,9,13 in SDF-1 40ug/kg group was beyond surgical group. After 8 weeks, MMP-13 mRNA expression in the SDF-1 40ug/kg group increase had not much change demonstrated that the model only stood on the middle stage of OA. Similarly, Wang, $G$ et research showed TN14003 can target blocking SDF-1/CXCR4 signaling pathway in vivo, reduce the expression and secretion of MMP-3, MMP-9, and MMP-13 in cartilage tissue, and reduce the degradation of collagen II and aggregating proteoglycan, thus delaying the degeneration of articular cartilage. [28]

Col II and ACAN are the principal component of cartilage ECM, and their expression and apoptosis are the best indicators of cartilage degeneration. [20] In our study, the alteration of Col II and ACAN mRNA and protein was downregulated as a result of SDF-1 injected increase of concentration and time. In SDF-1 $40 \mathrm{ug} / \mathrm{kg}$ injected group the degradation of Col II and ACAN after eight weeks were more than the surgical group. Compared with classical surgical method, the loss of COL $\square$ ACAN is more, and the time of building model are shorter. Other researchers also obtained the similar result as our experiment. By changing the content of SDF-1 in the joint, SDF-1/CXCR4 signaling pathway can be regulated, which affects the apoptosis of chondrocyte, and inflammation, and matrix Catabolism [20, 28-30] 
The results of current research were all highly consistent with the predictions that exogenous SDF- 1 intraarticular injection could establish the OA rabbit model. High dose of SDF-1 40ug/kg can cause the degeneration of rabbit knee joints faster, reaching middle stage of $O A$, and the efficacy similar to traditional surgical modeling. All rabbits were alive, no adverse reactions and complications of joint such as infection and instability occurred in surgical modeling. [31, 32] This provides a new method for modeling animals knee joint. It is more beneficial to the research of pathophysiology of human knee OA.

Some limitations remain in the present study. Firstly, only 12 weeks were observed in the current study, and the pathological features at the early and middle stages of OA were mainly presented in our model. Whether the model is applicable to the later stage of OA still needs a longer period of observation. Secondly, OA is a complex disease and the pathological changes of OA are the result of many cytokines and chemokines synergistic effect. Only SDF-1 was used to induce modeling, which may ignore the influence of other factors. Thirdly, this experiment has not been compared with other chemical modeling methods, and cannot fully explain the advantages of the same type of modeling methods. Further studies of SDF-1 induced OA model will be carried out to overcome above shortages in the future.

\section{Conclusion}

The present study demonstrated that intra-articular injection of SDF-1 can induce the release of inflammatory cytokines, MMPs secretion increased, the inhibition of ACAN, COL $₫$ synthesis, and accelerated extracellular matrix degradation. SDF-1 40ug/kg injection three times for 12 weeks may be an appropriate approach to induce $\mathrm{OA}$ rabbit knee model more rapidly and easily than traditional surgical modeling.

\section{Materials And Methods}

Animals and reagents.

All male 3-month-old Japanese white rabbits (weighting 2-2.5 kg) were obtained from the Experimental Animal Department of Kunming Medical University (Kunming, China) and were adapted for one week prior to the experimental procedures. The Institutional Ethics Committee of Kunming Medical University approved all experimental protocols, using an animal model. All surgical procedures were performed in accordance with Guidelines for the ethical review of laboratory animal welfare People's Republic of China National Standard GB/T 35892 - 2018 (Issued 6 February 2018 Effective from 1 September 2018).

The SDF - 1 alpha human species ,1000ug/ml, which was purchased from Pepro Tech Co., Ltd. (Rocky Hill, America) used in the present experiment was diluted to different concentrations mixing with normal saline. The 3,3'-diaminobenzidine (DAB) Substrate kit was purchased from ZSGB-Bio Co., Ltd. (Beijing, China), and Hematoxylin-Eosin/HE Staining Kit was purchased from Boster Biological Technology Co. Ltd. (Wuhan, China).

Rabbit OA model and SDF-1 treatment. 
Forty-five rabbits were randomly allocated into five groups: Normal saline (blank group), SDF-1 low level (10ug/kg group), SDF-1 medium level (20ug/kg group) and SDF-1 high level (40ug/kg group),surgery (control group). The knees of blank group and SDF-1 group rabbits' right hind legs were injected with $0.2 \mathrm{ml}$ volumes of saline or various concentrations of SDF-1, three times a week interval of one day. Surgery group was performed with classical surgical modeling method, Hulth method, which cut off medial collateral ligament, anterior cruciate ligament, and posterior cruciate ligament of the knee joint were broken and removes medial meniscus. Penicillin $(20,000 \mathrm{U} / \mathrm{kg})$ was injected daily for three days after operation to prevent infection. [33] All experimental rabbits were fed normally and motivated limb movements. Throughout the modeling process the behavior and weight changes of rabbits were observed. Four, eight, twelve weeks after operation, three rabbits from every group were sacrificed under intravenous anesthesia each time, and the sample was collected from experimental knees.

\section{Macroscopic observation and histological assessment}

After sacrifice, right knee joints were opened and the gross appearance of the femoral condyle was assessed and photographed. Osteochondral samples were collected from the intermediate region of the medial femoral condyles and fixed with $4 \%$ neutral formalin for $48 \mathrm{~h}$, decalcified with $14 \%$ EDTA (Hyclone Co., Ltd. ,Logan, Utah, America) for 15 days, dehydrated through a series of graded ethanol solutions, cleared in xylene, and embedded in paraffin. Sections, $5 \mu \mathrm{m}$ in thickness, were stained with hematoxylineosin (H\&E) to examine tissue morphology. Histological samples were graded using a modified Mankin's score in table 1.

\begin{tabular}{ccc}
\hline Item & Grade/classification & Grade \\
\hline Structural intergrity & Normal & 0 \\
& Irregular Surface & 1 \\
& Pannus formation & 2 \\
& Fissures into transitional layer & 3 \\
& Fissures into emmiting layer & 4 \\
& Fissures into calcified layer & 5 \\
Cells & Complete disorganization & 6 \\
& Normal & 0 \\
& Hypercellularity & 1 \\
Hematoxylin-eosin staining & Cloning & 2 \\
& Hypocellularity & 3 \\
& Normal & 0 \\
& Slight reduction & 1 \\
& Moderate reduction & 2 \\
& Severe reduction & 3 \\
& No dye noted & 4 \\
& Normal & 0 \\
& Disruption & 1 \\
\hline
\end{tabular}

Table 1. modified Mankin score (criteria for histological evaluation) 
After rehydrating with PBS, deparaffinized sections were quenched endogenous peroxidase activity with $\mathrm{H} 2 \mathrm{O} 2$ for immunological staining. Then sections were blocked with $5 \%$ normal goat serum (Bioss, Beijing, China) in PBS and incubated with primary antibodies against IL-1 (Bioss, Beijing, China) and TNF-a (Bioss, Beijing, China) overnight at $4^{\circ} \mathrm{C}$. The osteochondral were incubated with the second antibody and biotin-labeled horseradish peroxidase after incubation overnight at $4{ }^{\circ} \mathrm{C}$ with the primary antibody (1:200). Subsequently, the antibody binding was visualized with a 3, 3'-diaminobenzidine tetrahydrochloride (DAB) kit (Boster, China) before brief counterstaining with hematoxylin. Finally, Staining results were evaluated using light microscopy (Nikon, Japanese).

\section{ELISA}

Before dissecting knee joint, the synovial fluid was extracted with a $1 \mathrm{~mL}$ syringe and put into a $1 \mathrm{~mL}$ centrifuge tube frozen and stored. The levels of MMP-3, 9, 13 in synovial fluid were measured by ELISA according to the ELISA kit (R\&D Systems, Minnesota, USA) manufacturer's instructions. Absorbance at a wavelength of $450 \mathrm{~nm}$ was detected using a UV-2600 microplate reader (Shimadzu Corporation, Kyoto, Japan).

Real-time quantitative reverse transcription-polymerase chain reaction (qRT-PCR) analysis To evaluate the effect of SDF-1 on OA chondrocytes, the expressions of arthritis-related genes and cartilage-related genes. MMP-3, 9, 13, aggrecan, type II collagen mRNA expression of OA cartilage was detected by qRT-PCR. For the in vivo study, cartilage collected at 4, 8 and 12 weeks was pulverized in liquid nitrogen. TRIZOL reagent (Lifetech Limited, USA) was added to lyse the cells. Chondrocytes were washed once with PBS and the total RNA was extracted using a RNA isolation kit (Lifetech Limited, USA) according to the manufacturer's instructions. Using a reverse transcription kit (Fermentas Company, USA), approximately $2 \mu \mathrm{g}$ total RNA was used as a template and reverse transcribed into cDNA. The qRT-PCR reactions were carried out using a Quantitative PCR Detection System (Realplex 4, Eppendorf Corporation, USA) with FastStart Universal SYBR Green Master (Kapa Biosystems, Boston, USA). PCR thermocycling conditions were as follows: $10 \mathrm{~min}$ at $95^{\circ} \mathrm{C}, 15 \mathrm{~s}$ at $95^{\circ} \mathrm{C}$ and $30 \mathrm{~s}$ at $60^{\circ} \mathrm{C}$. The primers used for PCR are shown in Table 2. RNA expression levels were calculated by using the $2-\Delta \Delta \mathrm{Ct}$ method and glyceraldehyde-3-phosphate dehydrogenase (GAPDH) served as the internal reference. 


\begin{tabular}{ccc}
\hline Name & Sequence & Length \\
\hline GAPDH-F & TTAACTCTGGCAAAGTGGAT & 89 \\
GAPDH-R & GGTGGAATCATACTGGAACA & \\
MMP3-F & CCAGACATATAGCAGAAGAC & 106 \\
MMP3-R & AACTCCGACTGTGAAGAT & \\
MMP9-F & TCTGCAACGTGAATGTCT & 75 \\
MMP9-R & AGTACCTCCCATCCTTGA & 81 \\
MMP13-F & AACAGTAACGAGGATGAT & \\
MMP13-R & CAGAGGATGGTAGTATGAT & 100 \\
Collagen II-F & AGAGGTATAATGATAAGGAT & \\
Collagen II-R & GTGTCTTCACAGATTATG & 86 \\
Aggrecan-F & GTAGTGGTGAATCTTCTG & \\
Aggrecan-R & TAAGCCTTCTTCTTCTCT &
\end{tabular}

Table 2. Sequences of polymerase chain reaction primers used for the detection of miRNA expression.

\section{Western blot analysis}

Protein expression levels of type II collagen and aggrecan were measured by western blot, which are the main components of the extracellular matrix of articular cartilage. To prepare total protein from cartilage tissues obtained at 4, 8 or 12 weeks after treatment. The bicinchoninic acid assay method was used to assess the protein quantity and quality. Protein samples $(80 \mu \mathrm{g})$ were separated by SDS-polyacrylamide gel (3 h) and transferred to polyvinylidene difluoride membrane (wet method, $200 \mathrm{~mA}$ current for $60 \mathrm{~min}$ ). PVDF membranes were blocked in $5 \%$ skimmed milk powder for $1 \mathrm{~h}$, followed by incubating with primary antibodies (Abcam, Cambridge, UK; anti-Col II at 1:1000, anti-aggrecan at 1:1000 or anti-ßactin at 1:5000) overnight at $4^{\circ} \mathrm{C}$. After washing three times with TBST, the membranes were incubated with secondary antibodies (Abcam, Cambridge, UK; anti-mouse or anti-rabbit; dilution, 1:8,000) for $2 \mathrm{~h}$ at room temperature. Subsequent to TBST washing three more times, the ECL reagent (Thermo Fisher Scientifc, Inc.) was added for 1-3 min incubation in the dark. The band intensities were quantified by densitometry with Image J 1.46r software (National Institutes of Health, Bethesda, MD, USA).

\section{Statistical analysis}

All the data are presented as the mean \pm standard deviation. Differences between two groups were analyzed by unpaired t-test or the Mann-Whitney $U$ test for experiments in which the datasets were not normally distributed. Differences in multiple groups were analyzed by ANOVA. GraphPad Prism 6 software (GraphPad Software, La Jolla, CA, USA) was used for all statistical analyses. P values $<0.05$ were considered statistically significant. All experiments were repeated at least three times. 


\section{Abbreviations}

$\mathrm{OA}$

Osteoarthritis; SDF-1:Stromal cell-derived factor 1;

CXCR4

C-X-C chemokine receptor type 4; MMPs:matrix metalloproteinases

HE

Hematoxylin-eosin; DAB:3,3'-diaminobenzidine tetrahydrochloride;

COL $\triangle$

Type II collagen; ACAN:aggrecan

\section{Declarations}

\section{Ethics approval and consent to participate}

The present study was approved by the Institutional Ethics Committee of Kunming Medical University.

The reference number: kmmu2020416.

\section{Consent for publication}

Not applicable.

\section{Availability of data and material}

All data generated or analysed during this study are included in this published article.

\section{Competing interests}

The authors declare that they have no competing interests.

\section{Authors' contributions}

YLL designed the study. CZL and YHH performed the research and wrote the paper. GLW and DJL analyzed data. GFC and $\mathrm{CH}$ collated the data and checked the results. All authors read and approved the final manuscript.

\section{Funding}

This study was supported by the National Natural Science Foundation of China [No. 81960409, No.81760403]; Yunnan Key projects of the Natural Science Foundation [No.2017FE467(-007)]; the Yunnan Province Medical Leaders Talent Project (No.L-201601) and Expert workstation project of Shiyi Chen [No.2018IC102]. The funders had no role in study design, data collection and analysis, decision to publish, or preparation of the manuscript. 


\section{Author details}

1 Departments of Sports Medicine, The First Affiliated Hospital, Kunming Medical University, Kunming, Yunnan 650032, P.R. China.

\section{References}

1. Joseph E. Pizzorno ND, Michael T, Murray ND, Herb Joiner-Bey ND, in The Clinician's Handbook of Natural Medicine (Third Edition), 2016

2. Wang Huan, Sun He, Zhang Yaonan, Wang Fei, Xue Qingyun. Morbidity of primary knee osteoarthritis in different compartments in Chinese population aged over 40 years old. Chinese Journal of Bone and Joint Surgery Vol.12, No.7, Jul. 2019

3. Felson DT: Epidemiology of hip and knee osteoarthritis. Epidemiol Rev 1988;10:1-28.

4. Cope, PJ; Ourradi, K; Li, Y; et al. Models of osteoarthritis: the good, the bad and the promising. [J].Osteoarthritis Cartilage.2019,27(2):230-239

5. McCoy, A. M. Animal Models of Osteoarthritis: Comparisons and Key Considerations. Veterinary Pathology. 52 (5), 803-818 (2015)

6. Lampropoulou-Adamidou K, Lelovas P, Karadimas EV, Liakou C, Triantafillopoulos IK, Dontas I, et al. Useful animal models for the research of osteoarthritis[J]. Eur J Orthop Surg Traumatol. 2014,24(3):263-71.

7. Rogart J N, Barrach H J,. CCO. Articular collagen degradation in the Hulth-Telhag model of osteoarthritis.[J]. Osteoarthritis \& Cartilage. 1999,7(6):539-47.

8. Park, J; Lee, J; Kim, Kl; et al. A Pathophysiological Validation of Collagenase II-Induced Biochemical Osteoarthritis Animal Model in Rabbit.[J].Tissue Eng Regen Med.2018,15(4):437-444

9. Kanbe K, Takagishi K, Chen Q. Stimulation of matrix metalloprotease 3 release from human chondrocytes by the interaction of stromal cell-derived factor 1 and CXC chemokine receptor 4 . Arthritis Rheum 2002; 46:130-137. PMID: 11817585

10. Kanbe K, Takemura T, Takeuchi K, Chen Q, Takagishi K, Inoue K. Synovectomy reduces stromalcellderived factor-1 (SDF-1) which is involved in the destruction of cartilage in osteoarthritis and rheumatoid arthritis. J Bone Joint Surg Br. 2004; 86(2):296-300. PMID: 15046450

11. Wei F, Moore DC, Wei L, Li Y, Zhang G, Wei X, et al. Attenuation of osteoarthritis via blockade of the SDF-1/CXCR4 signaling pathway[J]. Arthritis Res Ther. 2012,14(4):R177.

12. Jia, D; Li, Y; Han, R; et al. miR-146a-5p expression is upregulated by the CXCR4 antagonist TN14003 and attenuates SDF-1 induced cartilage degradation. [J].Mol Med Rep.2019,19(5):4388-4400

13. Q. Xu, X.-C. Sun, X.-P. Shang, and H.-S. Jiang, "Association of CXCL12 levels in synovial fluid with the radiographic severity of knee osteoarthritis," Journal of Investigative Medicine, vol. 60, no. 6, pp. 898-901, 2012. 
14. X. Y. Wang, Y. Chen, X. J. Tang, L. H. Jiang, and P. Ji, "AMD3100 attenuates matrix metalloprotease-3 and -9 expressions and prevents cartilage degradation in a monosodium iodo-acetate-induced rat model of temporomandibular osteoarthritis," Journal of Oral and Maxillofacial Surgery, vol. 74, no. 5, pp. 927.e1-927.e13, 2016

15. Moody, HR; Heard, BJ; Frank, CB; et al. Investigating the potential value of individual parameters of histological grading systems in a sheep model of cartilage damage: the Modified Mankin method. [J]. J Anat.2012,221(1):47-54

16. Sato T, Konomi K, Yamasaki S, Aratani S, Tsuchimochi K, Yokouchi M, et al. Comparative analysis of gene expression profiles in intact and damaged regions of human osteoarthritic cartilage[J]. Arthritis Rheum. 2006,54(3):808-17.

17. Little C, Smith M. Animal Models of Osteoarthritis. Current Rheumatology Reviews.293 2008;4(3).

18. Xu, J; Yan, L; Yan, B; et al. Osteoarthritis Pain Model Induced by Intra-Articular Injection of Monolodoacetate in Rats. [J]. J Vis Exp.2020,(159):

19. Cott, $\mathrm{CEH}$; Holland, G; Krahelski, O; et al. Patterns of cartilage loss and anterior cruciate ligament status in end-stage osteoarthritis of the knee. [J].Bone Joint J.2020,102-B(6):716-726

20. Wang, K; Li, Y; Han, R; et al. T140 blocks the SDF-1/CXCR4 signaling pathway and prevents cartilage degeneration in an osteoarthritis disease model. [J]. PLoS One.2017,12(4):e0176048

21. Tchetina, EV; Glemba, KE; Markova, GA; et al. Development of Postoperative Pain in Patients with End-Stage Knee Osteoarthritis Is Associated with Upregulation of Genes Related to Extracellular Matrix Degradation, Inflammation, and Apoptosis Measured in the Peripheral Blood before Knee Surgery.[J].Life (Basel).2020,10(10):

22. Zhao, J; Duan, L; Wang, R; et al. Roflumilast prevents lymphotoxin a (TNF- $\beta$ )-induced inflammation activation and degradation of type 2 collagen in chondrocytes.[J].Inflamm Res.2020,():

23. Wang, MN; Liu, L; Zhao, LP; et al. [Research of inflammatory factors and signaling pathways in knee osteoarthritis]. [J].Zhongguo Gu Shang.2020,33(4):388-92

24. Chu XQ, Wang JJ, Dou LD, Zhao G. Cartilage oligomeric matrix protein and matrix metalloproteinase3 expression in the serum and joint fluid of a reversible osteoarthritis rabbit model[J]. Genet Mol Res. 2015,14(4):14207-15.

25. Nam SI, Kwon TK. Dexamethasone inhibits interleukin-1 beta-induced matrix metalloproteinase-9 expression in cochlear cells[J]. Clin Exp Otorhinolaryngol. 2014,7(3):175-80.

26. Nugent M. MicroRNAs: exploring new horizons in osteoarthritis[J]. Osteoarthritis Cartilage. 2016,24(4):573-80.

27. Mittal R, Patel AP, Debs LH, Nguyen D, Patel K, Grati M, et al. Intricate Functions of Matrix Metalloproteinases in Physiological and Pathological Conditions[J]. J Cell Physiol. 2016,231(12):2599-621.

28. Wang, G; Li, Y; Meng, X; et al. The study of targeted blocking SDF-1/CXCR4 signaling pathway with three antagonists on MMPs, type II collagen, and aggrecan levels in articular cartilage of guinea pigs. [J].J Orthop Surg Res.2020,15(1):195 
29. Xiang, Y; Li, Y; Yang, L; et al. miR-142-5p as a CXCR4-Targeted MicroRNA Attenuates SDF-1-Induced Chondrocyte Apoptosis and Cartilage Degradation via Inactivating MAPK Signaling Pathway. [J]. Biochem Res Int.2020,2020):4508108

30. Bragg, R; Gilbert, W; Elmansi, AM; et al. Stromal cell-derived factor-1 as a potential therapeutic target for osteoarthritis and rheumatoid arthritis. [J]. Ther Adv Chronic Dis.2019,10():2040622319882531

31. Arunakul, M; Tochigi, Y; Goetz, JE; et al. Replication of chronic abnormal cartilage loading by medial meniscus destabilization for modeling osteoarthritis in the rabbit knee in vivo. [J].J Orthop Res.2013,31(10):1555-60

32. Pauly, HM; Larson, BE; Coatney, GA; et al.Assessment of cortical and trabecular bone changes in two models of post-traumatic osteoarthritis.[J].J Orthop Res.2015,33(12):1835-45

33. Rogart, JN; Barrach, $\mathrm{HJ}$; Chichester, $\mathrm{CO}$; Articular collagen degradation in the Hulth-Telhag model of osteoarthritis. [J]. Osteoarthritis Cartilage.1999,7(6):539-47

\section{Figures}

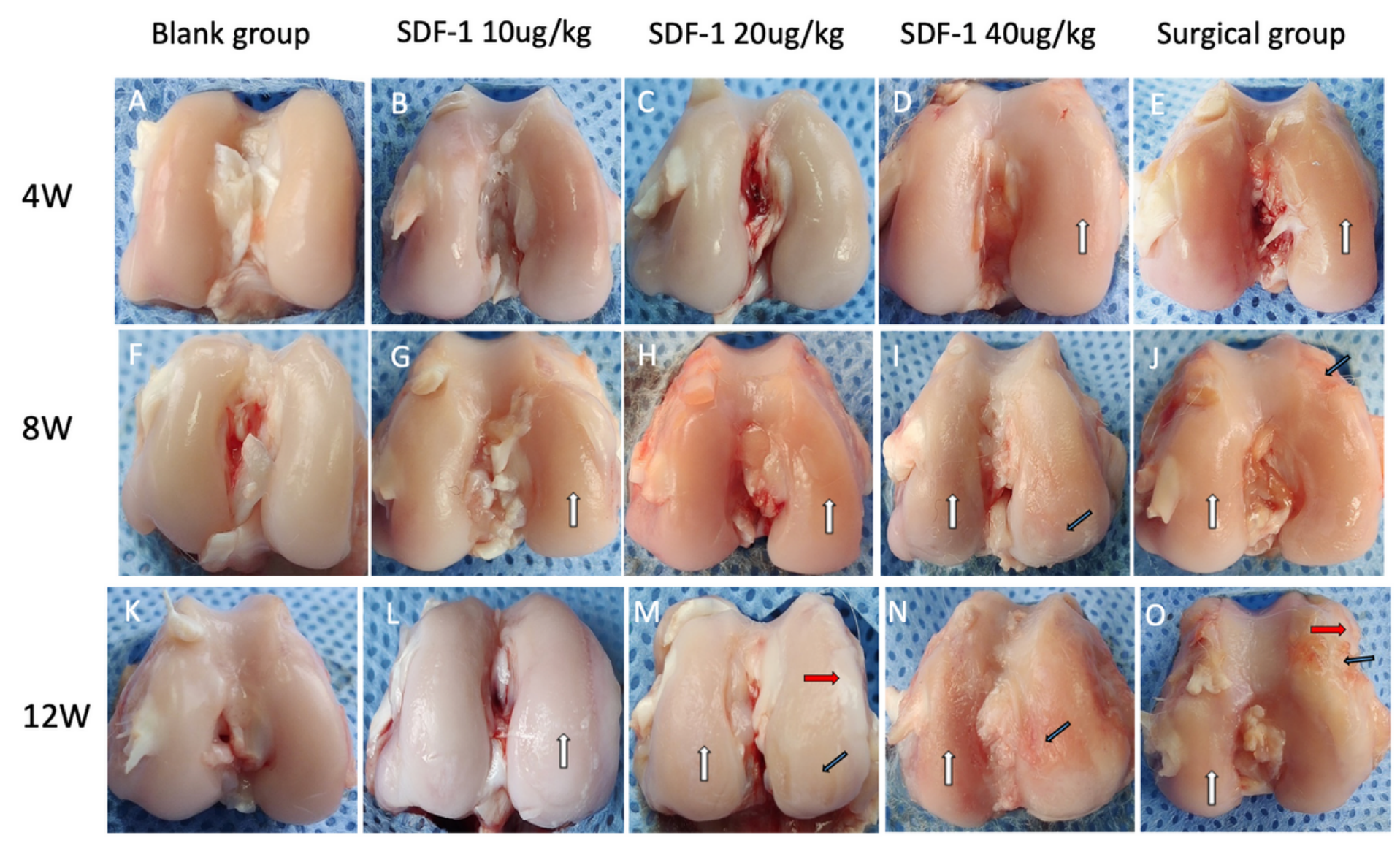

\section{Figure 1}

Macroscopic observation of cartilage specimens 4,8,12 weeks later after intra-articular injection of SDF-1 and surgery. A,F,K saline injection knees; B-D,G-I,L-N various concentrations of SDF-1 injection knees; E,J,O 
surgical knee. White arrows $₫$ Dry and roughness of the cartilage surface. Black arrows: Cartilage defection. Red arrows: Osteophytes formed at the joint margins

A
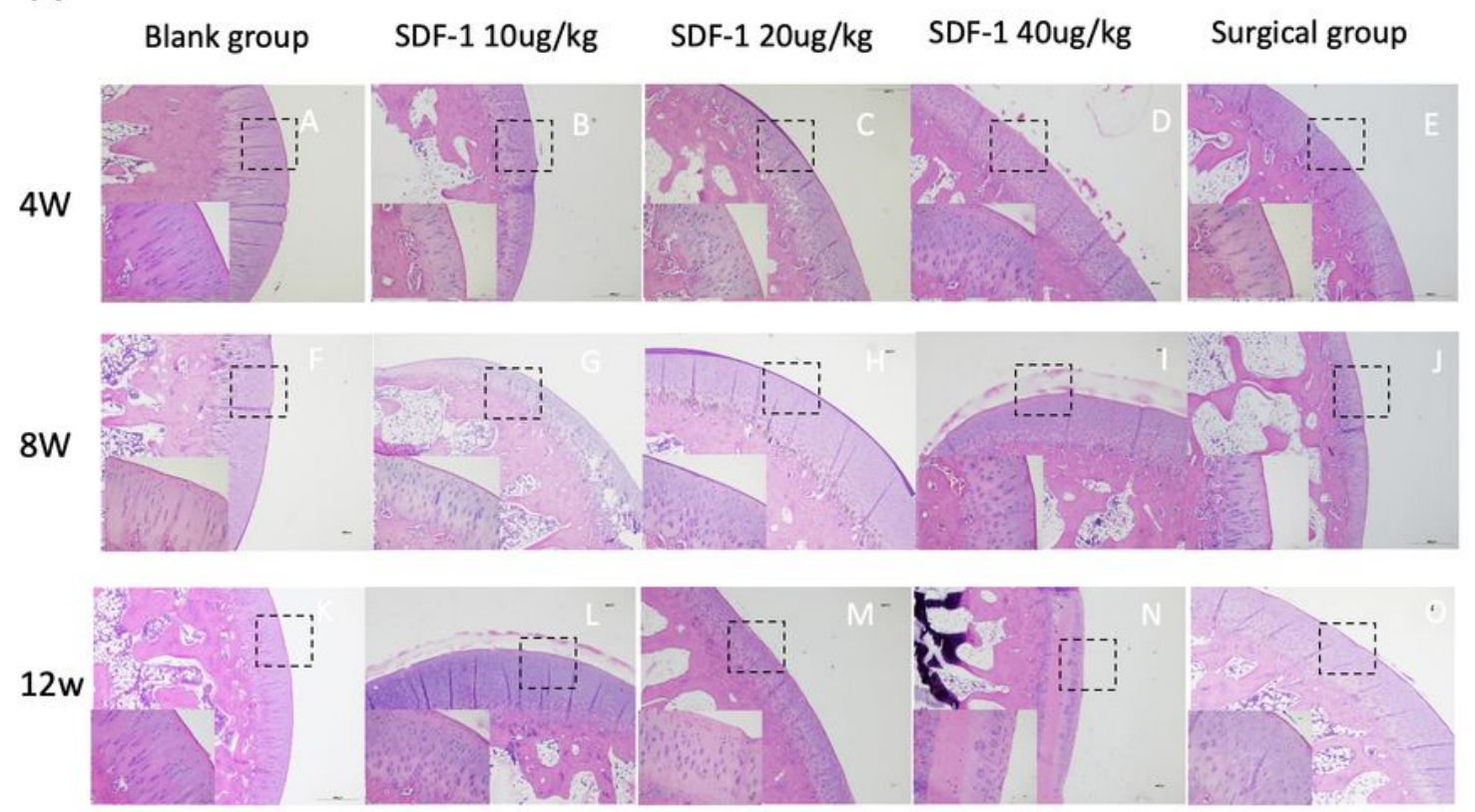

B

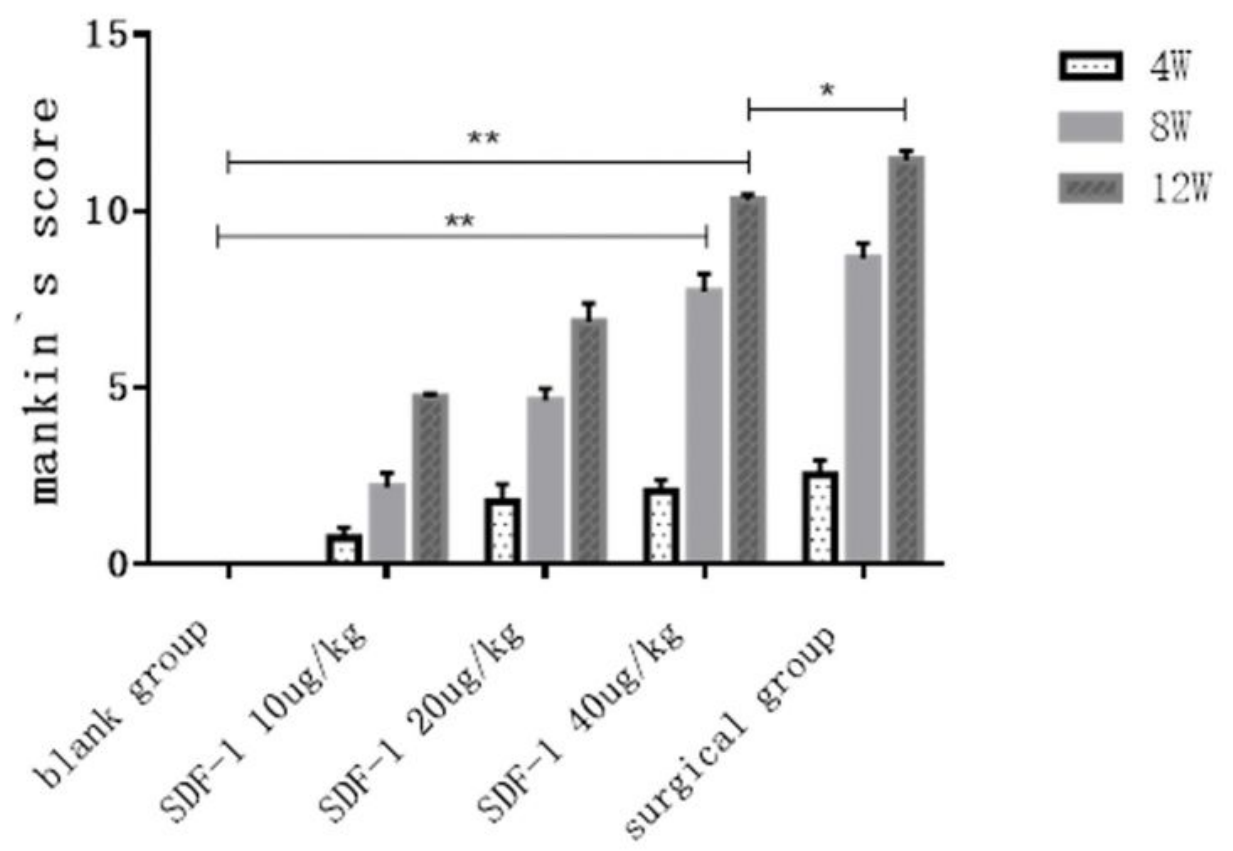

Figure 2

A Hematoxylin-eosin staining of osteochondral specimens from the intermediate region of the medial femoral condyle after injection and surgery. A,F,K Normal saline injected knees; B-D,G-I,L-N SDF-1 injected 
knees; E,J,O surgical knee. 40x magnification. B The modified Mankin's scores. Data were measured as mean \pm standard error of the mean. ( ${ }^{*} p<0.05,{ }^{*} p<0.01$ and ${ }^{* *} p<0.001$ vs. control values).

A
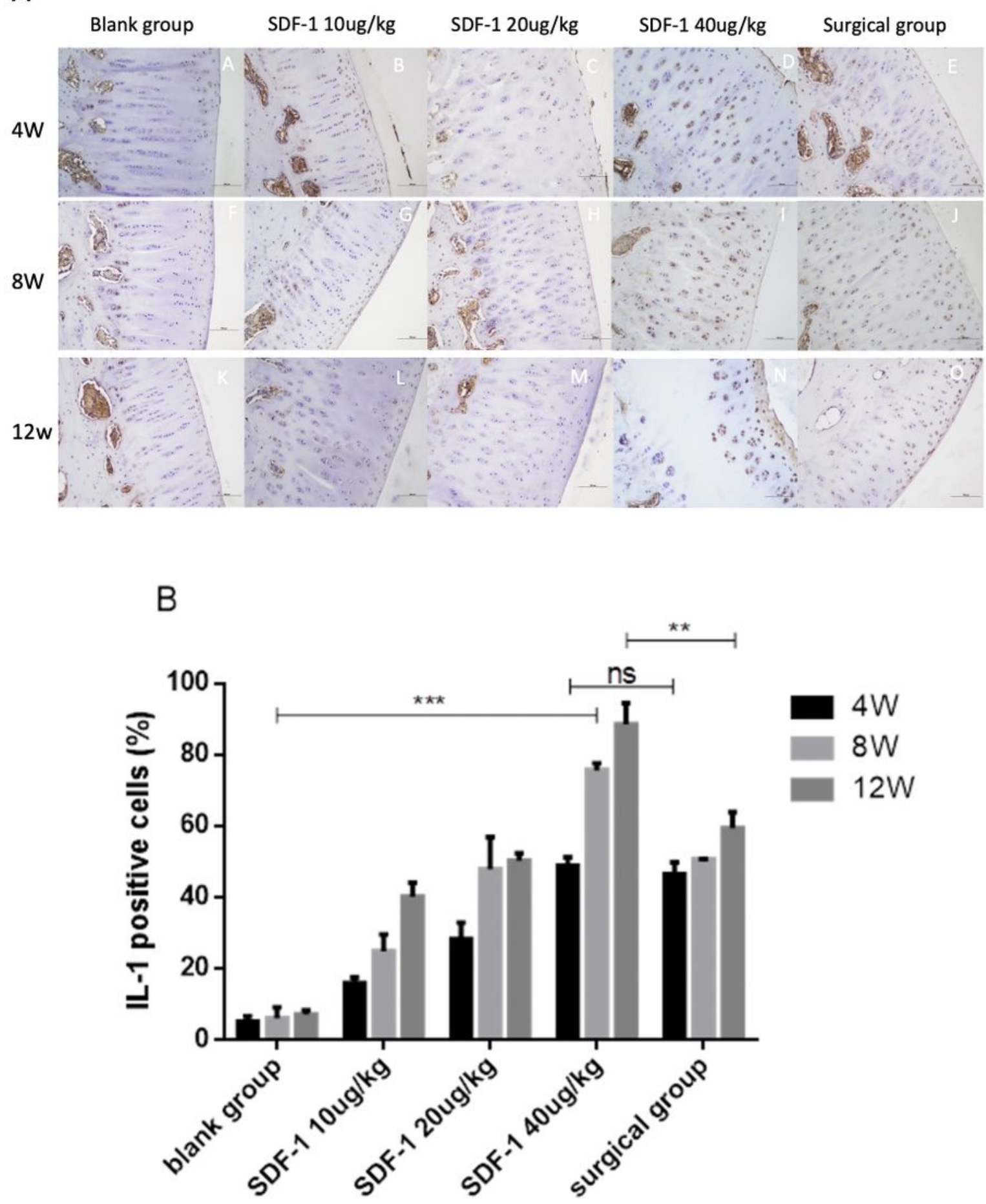

Figure 3

A IL-1 staining of osteochondral specimens from the intermediate region of the medial femoral condyle after immobilization. A,F,K Normal saline injected knees; B-D,G-I,L-N SDF-1 injected knees; E,J,O surgical knee. 40× magnification. B The percentages of IL-1 positive cells were randomly counted using three 
fields. Data were measured as mean \pm standard error of the mean. $\left({ }^{*} p<0.05,{ }^{*} p<0.01\right.$ and ${ }^{* * *} p<0.001$, NS: no significant difference).
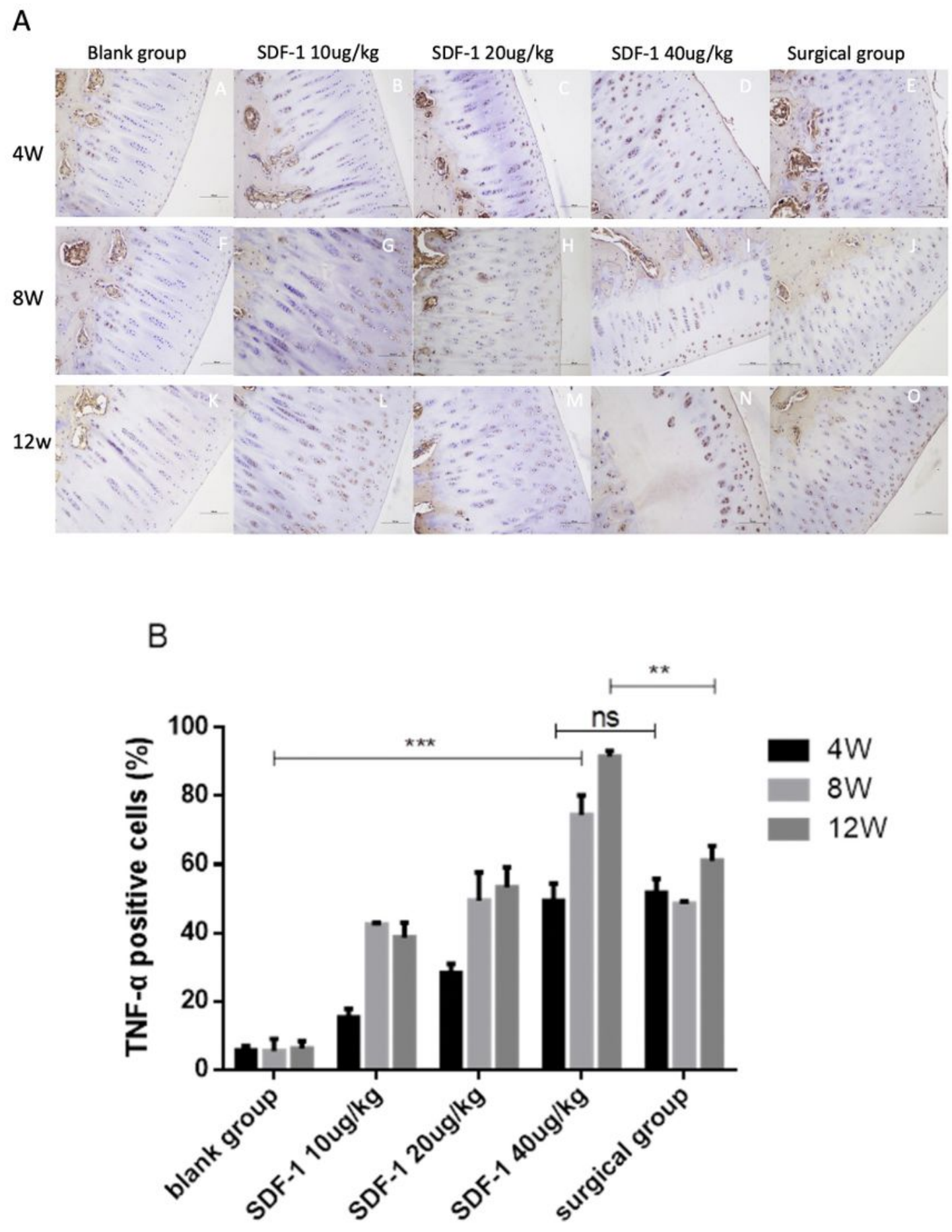

Figure 4

A TNF-a staining of osteochondral specimens from the intermediate region of the medial femoral condyle after immobilization. A,F,K Normal saline injected knees; B-D,G-I,L-N SDF-1 injected knees; E,J,O surgical knee. 40x magnification. B The percentages of TNF-a positive cells were randomly counted using three 
fields. Data were measured as mean \pm standard error of the mean. $\left({ }^{*} p<0.05,{ }^{*} p<0.01\right.$ and ${ }^{* * *} p<0.001$, NS: no significant difference).
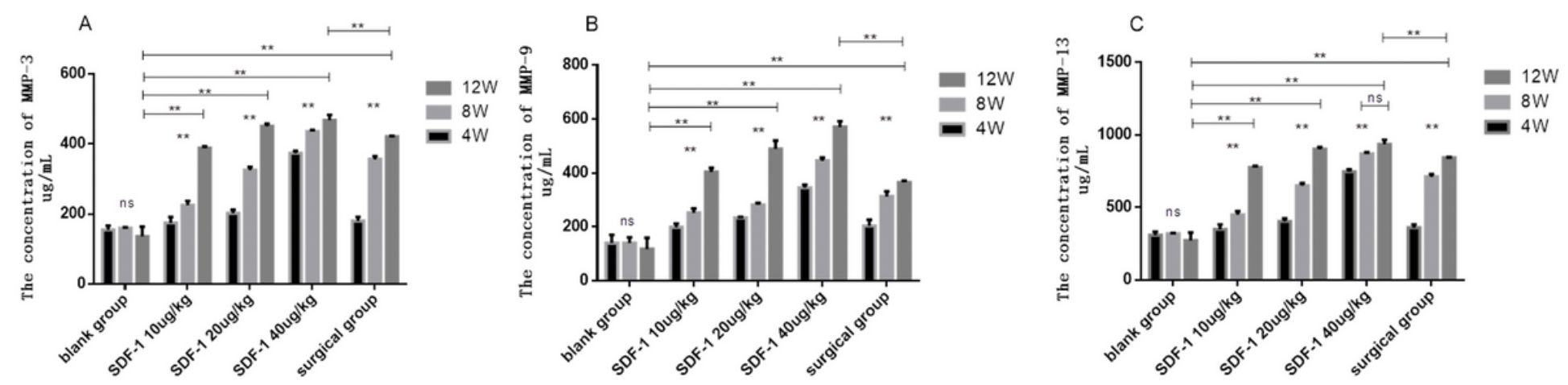

Figure 5

ELISA assay showed the MMPs level in the synovial fluid of the normal saline injected group, the SDF-1 injected group and the surgical group. A: MMP-3. B: MMPs-9. C: MMPs-13. Values are presented as mean $\pm S D .\left({ }^{*}<<0.05,{ }^{*} p<<0.01, n s p>0.01\right.$ no significant difference).
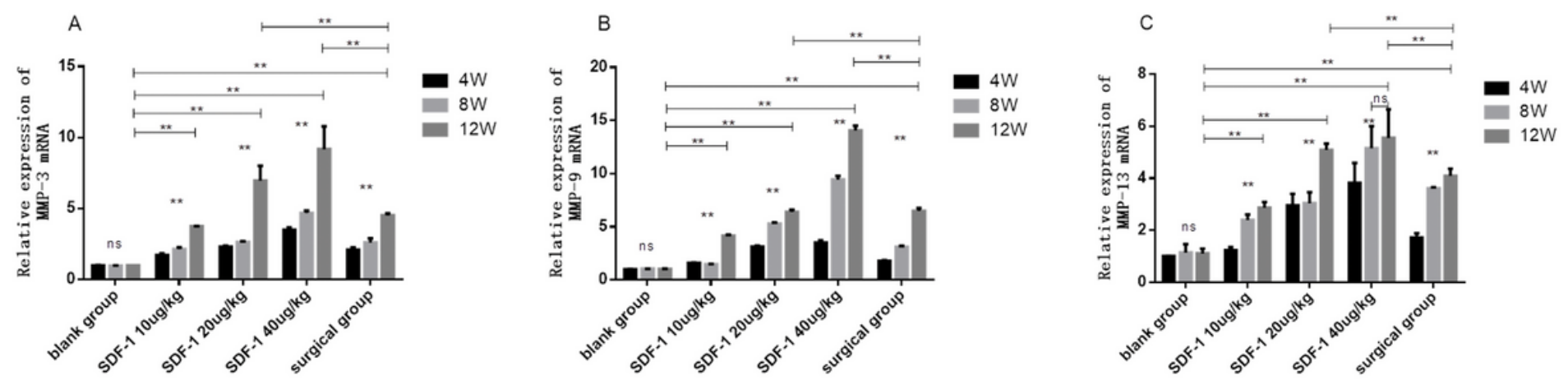

Figure 6

QRT-PCR was used to analyze the expression of MMPs mRNA in cartilage at 4, 8 and 12 weeks after treatment. A: MMP-3. B: MMP-9. C: MMP-13. Values are the means $\pm S D$. $\left({ }^{\star} p<0.05,{ }^{\star \star} p<0.01, n s p>0.01\right.$ no significant difference). 

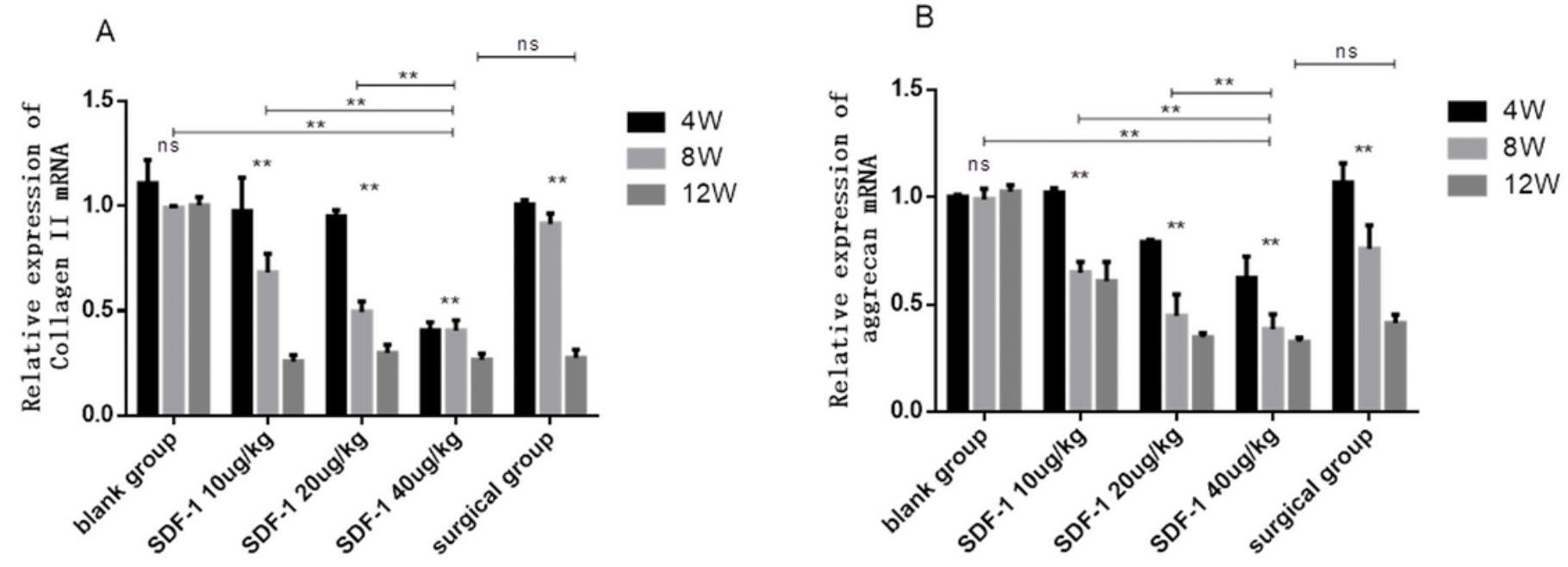

Figure 7

COL『mRNA and ACAN mRNA expression in chondrocytes at week 4,8 and 12 after the establishment of the OA model respectively. A: COLØmRNA. B: ACAN mRNA. Values are the means \pm SD. $\left({ }^{\star} p<0.05,{ }^{*} p<\right.$ 0.01 and $n s p>0.01$ no significant difference).

A

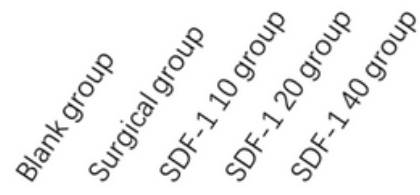

COL $\| 4 \mathrm{~W}$

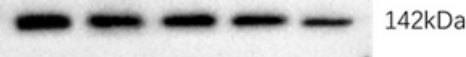

COL II 8W

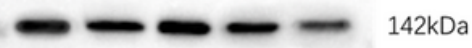

COL II $12 \mathrm{~W}$

$0-142 \mathrm{kDa}$

$\beta$-actin

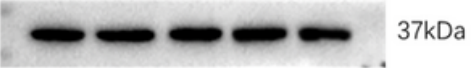

范

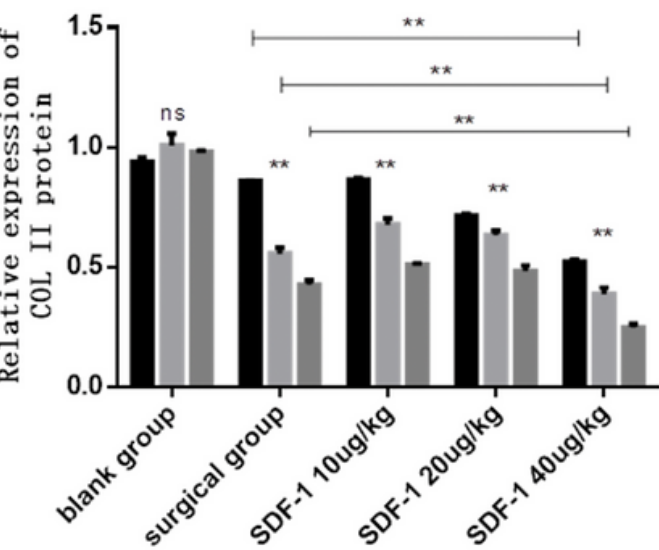

B

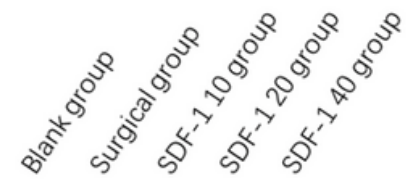

ACAN 4W

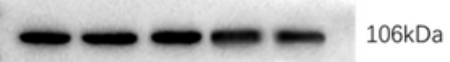

ACAN 8W

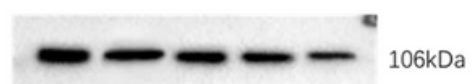

ACAN $12 \mathrm{~W}$

$106 \mathrm{kDa}$

$\beta$-actin
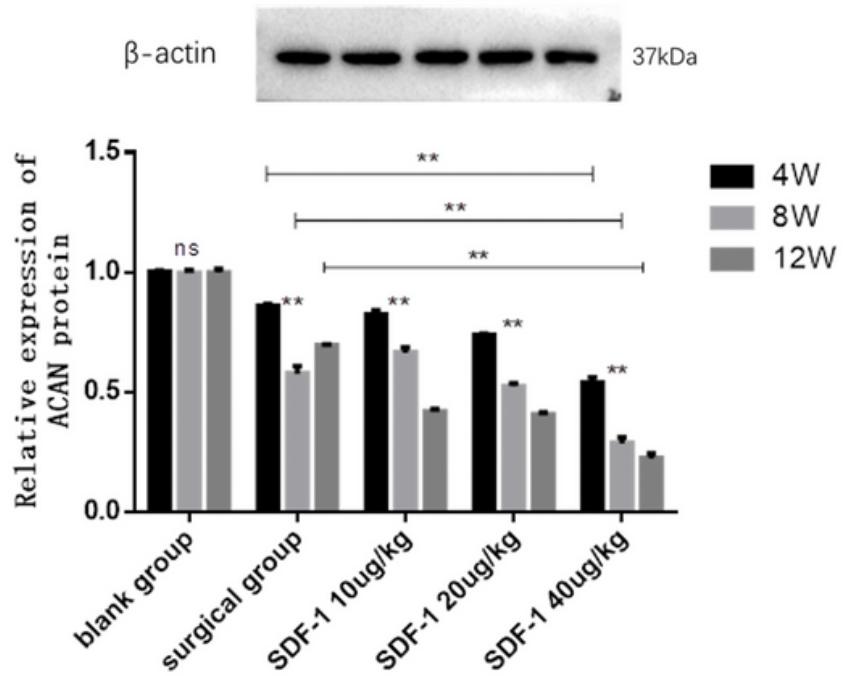


\section{Figure 8}

COL\and ACAN protein expressions were assessed in chondrocytes using western blotting. A:

COL\protein. B: ACAN protein. Data are presented as the mean \pm standard error of the mean. $\left({ }^{\star} p<0.05\right.$, ${ }^{* *} p<0.01$ and $n s p>0.01$ no significant difference). 